\title{
Adding value to scholarship in residency: Supporting and inspiring future emergency medicine research in Canada
}

\author{
Daniel K. Ting, MD*; Blair L. Bigham, MD, $\mathrm{MSc}^{\dagger}$; Shaun Mehta, MD; Ian Stiell, MD, MSc ${ }^{\S}$
}

\section{INTRODUCTION}

This is an exciting time for CFEM; it has recently conducted its first review and plans several changes to enhance the journal's impact and contributions to our field. ${ }^{1}$ CFEM faces the challenge of publishing highquality scholarly work while simultaneously providing opportunities for resident physicians to publish their work. ${ }^{1}$ Resident publication in peer-reviewed journals remains the gold standard for measuring scholarly output, and we recognize that, as the calibre of CFEM rises, so too must the calibre of academic output by the residents.

\section{THE CURRENT LANDSCAPE OF RESIDENT RESEARCH}

Residents face several barriers when aiming to publish rigorous, high-impact original research during dense and time-limited training programs. More than half of the Fellowship of the Royal College of Physicians (FRCP) training programs expect residents to submit a manuscript to a journal and an abstract to an international conference, ${ }^{2}$ which may unintentionally emphasize completion over quality, ${ }^{3}$ and encourage residents to "go through the motions" rather than conduct valuable work with true curiosity.

Given the time and resource constraints of resident projects, residents often choose traditional projects like case reports and retrospective work at their home centre with little expectation of producing high-impact scholarship and work alone. An approach where postgraduate training programs embrace a broader definition of scholarship and support the collaboration required to generate impactful science will encourage teamwork between residents across Canada. Removing requirements that encourage working in silos may allow for more impactful longitudinal or multicentre studies that span longer than a training period. We believe that such an approach would still develop the skills in research literacy required of modern clinicians, support residents on a more traditional research track, and lead to a more impactful scholarship for residents less inclined toward academic careers.

\section{BRINGING PURPOSE TO SCHOLARSHIP}

Scholarly activity is a fundamental part of all Canadian emergency medicine (EM) training programs. Beginning research training at this early stage helps build a foundation of success for future scholars, practitioners, and leaders. Historically, the focus of research has largely been in the basic or clinical sciences; however, the landscape of academia has changed significantly.

There has been an explosion of academic work in areas such as medical education, patient safety, quality improvement, and administration. These projects are instrumental in generating creative solutions to systems-level issues and should be encouraged to optimize the way we train physicians and deliver care. This shift is supported broadly, as evidenced by the updated 2015 definition of the CanMEDS scholar role, where emphasis is placed on developing structured critical appraisal skills. ${ }^{4}$ These principles were reinforced at the 2014 Canadian Association of Emergency

From the * Department of Emergency Medicine, University of British Columbia, Vancouver, BC; ${ }^{\dagger}$ Division of Emergency Medicine, McMaster University, Hamilton, ON; ${ }^{\ddagger}$ Division of Emergency Medicine, Department of Medicine, University of Toronto, Toronto, ON; and the ${ }^{\S}$ Department of Emergency Medicine, Ottawa Hospital Research Institute, University of Ottawa, Ottawa, ON.

Correspondence to: Daniel Ting, 2312 Pandosy St, Kelowna, BC V1Y 1T3, Canada; Email: daniel.ting@alumni.ubc.ca 
Physicians (CAEP) Academic Symposium, with scholarly work defined as a systematic approach to identify a problem and design a response. ${ }^{2}$

Trainees should pursue scholarship aligned with their career interests, instead of completing projects for the sake of requirement fulfillment. By welcoming a broad definition of scholarship, training programs can inspire a sense of ownership and meaning for trainees undertaking research projects. We urge institutions to recognize alternative forms of scholarship and to develop position statements, working groups, and discussion forums for less traditional albeit important topics.

\section{NURTURING RESEARCH PRODUCERS}

Although training programs have similar formal academic requirements, their scholarly output is uneven; further, some residents are more interested in academic undertakings than others, showing differing levels of engagement and productivity based on their career goals. ${ }^{2}$ Residents should be connected to mentors who are available and enthusiastic and who have scholarly experience in their area of interest. ${ }^{5}$ While some residents may not have a local mentor who fits these criteria, the rise of digital technologies overcomes traditional institutional and geographical barriers to scholarly collaboration. Academic gatherings, such as the CAEP Conference or the Network of Canadian Emergency Researchers (NCER) annual meeting, should be approached by attendees and organizers as an opportunity to spark academic networks. ${ }^{6}$ These collaborations can blossom in a "digital laboratory." Recently, some of these opportunities have taken on a formal structure, such as the CanadiEM Digital Scholars Fellowship for medical education. ${ }^{7}$

Furthermore, institutions should foster a culture that celebrates academic scholarship. Scholarly inquiry is an exercise in delayed gratification whose traditional rewards manifest relatively rarely and are bestowed to selective endeavors (e.g., conference presentation). Institutions can use social media as a simple and inexpensive strategy to increase the immediate impact of a broad range of scholarly work. For example, the University of Ottawa has recently integrated a Digital Scholarship and Knowledge Dissemination program ${ }^{8}$ within the structure of its academic department. This includes funded faculty positions and an institutional website to publish a large range of local scholarly work, from grand rounds to journal club summaries to article publications; such programs can be replicated more broadly.

\section{RESIDENT OPPORTUNITIES OFFERED BY CJEM}

CFEM created a new leadership team in November 2017, and this team recently affirmed a vision of "inspiring excellence in emergency medical care." We wish to be among the top three EM journals within 5 years. Does this mean fewer opportunities for learners at CFEM? Most certainly not. The CFEM editorial board recently approved this specific objective - "to provide scholarly opportunities for medical students, residents, and newly practicing physicians." The entire editorial team wishes to work with our younger authors to assist the development of their scholarship skills. Congratulations to Daniel Ting, a resident from University of British Columbia, who has nearly completed his term as our first "CFEM Intern." We welcome residents to apply for this internship position every year.

CFEM has many sections well suited to resident submissions. Clinical Correspondence (i.e., case reports, images) will be almost exclusively reserved for young Canadians. Resident Issues publishes articles written on subjects of importance to EM residents. Residents are welcome to submit a Commentary on other issues. fournal Club is a great opportunity because we expect these articles to be co-authored by a learner or new physician. Residents are frequently involved in Brief Educational Reports, submissions that discuss educational advances in EM. Finally, we have created a new category, Brief Original Research, comprising no more than 1,000 words and 15 references. This is well suited for smaller studies, some of which may have been resident research projects. Bottom line - CFEM loves publishing articles by residents!

Keywords: emergency medicine, medical education, residency

Acknowledgements: The authors thank Gerhard Dashi and Eddy Lang for their critical input in this manuscript.

Competing interests: None declared.

\section{REFERENCES}

1. Stiell I, Lang E, Atkinson P. A new chapter for CFEM. CFEM 2018;20(1):1-2. 
2. Calder LA, Abu-Laban RB, Artz JD, et al. CAEP 2014 Academic Symposium: How to make research succeed in your department: promoting excellence in Canadian emergency medicine resident research. CFEM 2015;17(5):591-9.

3. Ioannidis JP. Why most clinical research is not useful. PLoS Med 2016;13(6):e1002049.

4. Richardson D, Oswald A, Chan M-K, et al. Scholar. In: CanMEDS 2015 physician competency framework (eds. Frank JR, Snell L, Sherbino J). Ottawa: Royal College of Physicians and Surgeons of Canada; 2015, 24-25.

5. Gottlieb M, Fant A, King A, et al. One click away: digital mentorship in the modern era. Cureus 2017;9(11):e1838.
6. Gottlieb M, Sheehy M, Chan T. Number needed to meet: ten strategies for improving resident networking opportunities. Ann Emerg Med 2016;68(6):740-3.

7. Zaver F, Thomas A, Shahbaz S, et al. The CanadiEM Digital Scholars Program: an innovative international digital collaboration curriculum. C7EM 2017;19(S1):S32.

8. Rosenberg H. Development of EMOttawa's digital scholarship and knowledge dissemination program; 2018. Available at: https://emottawablog.com/2018/02/developmentof-emottawas-digital-scholarship-and-knowledge-disseminationprogram. 